\title{
SUFFICIENT CONDITION FOR BLACKHOLE FORMATION IN SPHERICAL GRAVITATIONAL COLLAPSE
}

\author{
ROBERTO GIAMBÓ, FABIO GIANNONI AND GIULIO MAGLI
}

\begin{abstract}
A sufficient condition for the validity of Cosmic Censorship in spherical gravitational collapse is formulated and proved. The condition relies on an attractive mathematical property of the apparent horizon, which holds if "minimal" requirements of physical reasonableness are satisfied by the matter model.
\end{abstract}

\section{INTRODUCTION}

Predicting the final state (blackhole or naked singularity) of the gravitational collapse of an initially regular matter distribution in General Relativity is still, to a large extent, an open problem, even in the special case of spherical symmetry. In fact, Penrose's "Cosmic Censorship" conjecture [1] stating that "every physically reasonable collapsing matter distribution forms a blackhole" seems to be overruled by several examples, in which what is likely to be a physically reasonable distribution of matter forms a visible null singularity (for instance in the dust and in the Vaydia case, see [2] and references therein) or even a timelike singularity, as in the case of gravitating clusters of rotating particles [3, , 7, 5, 6]. It is, therefore, necessary to obtain a rigorous formulation of censorship in mathematical terms, in order to be able to translate the conjecture in mathematically demonstrable assertions. In this formulation, the physics of the problem is obviously expected to play a fundamental role. For instance, the weak energy condition has naturally to be expected as one of the hypotheses of any cosmic censorship theorem.

In the present letter, we report on an investigation carried out recently devoted to the application of tecniques of non-linear o.d.e. to the censorship problem in spherical symmetry. The main result is a non-existence theorem for geodesics meeting the singularity in the past, which gives a sufficient condition for a spherically symmetric non-static metric to represent blackhole formation. The condition can be used as a test bed for probing models of collapse without knowing the explicit solution of the Einstein field equations.

\section{THE ANALYTICAL FRAMEWORK IN BRIEF}

We consider a spherically symmetric collapsing object in full generality. The matter model can thus be any model compatible with the weak energy condition. The general, spherically symmetric, non-static line element in comoving coordinates $t, r, \theta, \varphi$ can be written in terms of three functions $\nu, \lambda, R$ of $r$ and $t$ only as follows:

$$
\mathrm{d} s^{2}=-e^{2 \nu} \mathrm{d} t^{2}+e^{2 \lambda} \mathrm{d} r^{2}+R^{2}\left(\mathrm{~d} \theta^{2}+\sin ^{2} \theta \mathrm{d} \varphi^{2}\right),
$$

A fundamental quantity is the mass function $m(r, t)$ defined through

$$
1-\frac{2 m}{R}=g^{\mu \nu} \frac{\partial R}{\partial x^{\mu}} \frac{\partial R}{\partial x^{\nu}}
$$

that is

$$
m(r, t)=\frac{R}{2}\left[1-\left(R^{\prime}\right)^{2} e^{-2 \lambda}+(\dot{R})^{2} e^{-2 \nu}\right]
$$

where a dash and a dot denote derivatives with respect to $r$ and $t$ respectively. Field equations $G_{0}^{0}=8 \pi T_{0}^{0}$ and $G_{r}^{r}=8 \pi T_{r}^{r}$ relate derivatives of $m$ to the energy density $\epsilon$ and the radial stress $p_{r}$ of the material: 


$$
\begin{gathered}
m^{\prime}=4 \pi \epsilon R^{2} R^{\prime}, \\
\dot{m}=-4 \pi p_{r} R^{2} \dot{R},
\end{gathered}
$$

We consider only matter configurations admitting a regular center, and we suppose always that the collapse starts from regular initial data on a Cauchy surface $(t=0$, say), so that the singularities forming are a genuine outcome of the dynamics. If the solution is initially regular, (2.3) implies that the energy density - and therefore the spacetime - becomes singular whenever $R$ or $R^{\prime}$ vanish during the evolution. It follows, that singularities can be of two different kinds: shell crossing, at which $R^{\prime}$ vanishes while $R$ is non-zero, and shell focusing at which $R$ vanishes. The shell crossing singularities have been frequently considered as "weak" although no proof of extensibility is as yet available in the literature. In any case, in most physically interesting situations such singularities do not occur, so that we shall concentrate attention here only on the shell focusing case. Therefore, we assume that $R^{\prime}$ is initially non zero for non-zero $r$ and remains positive up to the formation of the focussing singularities.

The locus of the zeroes of the function $R(r, t)$ defines the singularity curve $t_{s}(r)$ by the relation $R\left(r, t_{s}(r)\right)=0$. Physically, $t_{s}(r)$ is the comoving time at which the shell of matter labeled by $r$ becomes singular. The singularity forming at $r=0, t=t_{s}(0)$ is called central as opposed to those occurring at $r=r_{0}>0, t=t_{s}\left(r_{0}\right)$.

A singularity cannot be naked if it occurs after the formation of the apparent horizon. The apparent horizon $\left(t_{h}(r)\right.$, say) is the boundary of the region of trapped surfaces and it is therefore defined, due to equation (2.1), by $R\left(r, t_{h}(r)\right)=2 m\left(r, t_{h}(r)\right)$. Since $R$ vanishes at a singularity, any naked singularity in spherical symmetry must be massless; at a massless singularity the horizon and the singularity form simultaneously. Since regularity of the center up to singularity formation requires $m(0, t)=0 \forall t<t_{s}(0)$, the center is always a candidate for nakedness. Other points ("non-central points") of the singularity curve can be naked only in presence of radial tensions. In fact, due to eq. (2.4), the mass is increasing in time in a collapsing $(\dot{R}<0)$ situation, if the radial pressure is positive [7].

To analyze the causal structure, observe that, if the singularity is visible to nearby observers, at least one outgoing null geodesic must exist, that meets the singularity in the past. Such a geodesic will be a solution of

$$
\frac{\mathrm{d} t(r)}{\mathrm{d} r}=\varphi(r, t)
$$

where

$$
\varphi(r, t):=\sqrt{-\frac{g_{r r}}{g_{00}}}=e^{\lambda-\nu}
$$

with initial datum $t\left(r_{0}\right)=t_{s}\left(r_{0}\right)$. For a problem of this kind, in which the initial point is singular (the function $\varphi$ is not defined at $\left(r_{0}, t_{s}\left(r_{0}\right)\right)$ ) no general results of existence/non existence are known. As a consequence, in the literature, an approach has been developed (see e.g. [2]) which makes use of l'Hospital theorem to identify the possible values of the tangent of the geodesic curve at the singularity. This is the approach that allowed a full understanding of the dust and of the Einstein cluster cases recalled above. However, to be successful, this tecnique requires complete integration of the field equations, a result which is far beyond our present understanding even in the simple case of the barotropic perfect fluid. On the other side, what actually enters the problem of the causal structure is only the function $\varphi$, not the whole solution of the field equations. Therefore, one can consider this problem as an existence/non-existence problem for the non-linear o.d.e. (2.5), in which the mathematical structure of the Einstein equations as well as the physics of the problem (like e.g. formation of trapped surfaces, weak energy condition) play a fundamental role. 


\section{THE NON-EXISTENCE THEOREM}

From now on we will assume the following condition on $p_{r}, \epsilon$ (see Remark 3.3 below):

$$
\epsilon>0, p_{r} \geq \operatorname{Max}\left\{-\epsilon,-\frac{1}{8 \pi R^{2}}\right\} .
$$

3.1. Theorem. If (3.1) holds and $\frac{\partial \varphi}{\partial t} \leq 0$ in a neighborhood of $\left(r_{0}, t_{s}\left(r_{0}\right)\right)$, with $r_{0} \geq 0$, the singularity forming at $\left(r_{0}, t_{s}\left(r_{0}\right)\right)$ is covered.

The theorem is based on a remarkable mathematical property of the apparent horizon, which we address in the following

3.2. Lemma. If (3.1) holds then there exists $r_{*}>r_{0}$ such that the apparent horizon $t_{h}(r)$ is a subsolution of (2.5) for $r \in\left(r_{0}, r_{*}\right)$.

Proof. Differentiating the equation $R\left(r, t_{h}(r)\right)=2 m\left(r, t_{h}(r)\right)$ with respect to $r$ we get

$$
\frac{\mathrm{d} t_{h}}{\mathrm{~d} r}=-\frac{2 m^{\prime}-R^{\prime}}{2 \dot{m}-\dot{R}}=-\Gamma \frac{R^{\prime}}{\dot{R}}
$$

where

$$
\Gamma:=\frac{1-8 \pi \epsilon R^{2}}{1+8 \pi p_{r} R^{2}}
$$

and the field equations (2.3)-(2.4) have been used. On the other end, from eq. (2.2) we get

$$
\varphi(r, t)=e^{\lambda-\nu}=-\left[1+e^{2 \nu} \dot{R}^{-2}\left(1-\frac{2 m}{R}\right)\right]^{-\frac{1}{2}} \frac{R^{\prime}}{\dot{R}}
$$

where a (crucial) minus sign is due to the fact that we consider a collapsing scenario (thus $\dot{R}$ is strictly negative, at least near the singularity). At $t=t_{h}$ the quantity in square brackets is equal to one so that $\varphi\left(r, t_{h}\right)=-R^{\prime} / \dot{R}$. It follows $d t_{h} / d r \leq \varphi\left(r, t_{h}\right)$ whenever the quantity $\Gamma$ is less than or equal to unity, that is

$$
\frac{8 \pi R^{2}\left(\epsilon+p_{r}\right)}{8 \pi p_{r} R^{2}+1}>0 .
$$

3.3. Remark. If $8 \pi p_{r} R^{2}+1$ is negative, the radial pressure would diverge to minus infinity at a singularity, a manifestly unphysical situation. Thus, we consider further only matter models satisfying $p_{r}>-1 / 8 \pi R^{2}$. This is a very weak bound; obviously it must be considered only if tensions are present, and at most it has to be extended to the whole of the collapsing object (in this case it suffices to require $p_{r}>-1 / 8 \pi R^{2}\left(r_{b}, t\right)$ where $r_{b}$ is the boundary of the object). Once this is satisfied, the inequality (3.2) holds if $\epsilon+p_{r}>0$.

Proof of Theorem 3.1. Let $t_{\rho}(r)$ the solution of $t^{\prime}(r)=\varphi(r, t(r))$ such that $t_{\rho}\left(r_{0}\right)=$ $t_{s}\left(r_{0}\right)$. By contradiction we suppose the existence of $r_{1}>0$ such that $t_{\rho}\left(r_{1}\right)<t_{h}\left(r_{1}\right)$ and $t_{\rho}(r) \leq t_{h}(r), \forall r \in\left[r_{0}, r_{1}\right]$. We can suppose $r_{1}<r_{*}$, where $r_{*}$ comes from Lemma 3.2 . Since $t_{\rho}\left(r_{0}\right)=t_{h}\left(r_{0}\right)$, one has

$$
\begin{aligned}
0<t_{h}\left(r_{1}\right)-t_{\rho}\left(r_{1}\right)=\left(t_{h}\left(r_{1}\right)-t_{\rho}\left(r_{1}\right)\right)-\left(\left(t_{h}\left(r_{0}\right)-t_{\rho}\left(r_{0}\right)\right)\right) & = \\
\left(t_{h}^{\prime}(\xi)-t_{\rho}^{\prime}(\xi)\right) r_{1} & =\left(t_{h}^{\prime}(\xi)-\varphi\left(\xi, t_{\rho}(\xi)\right)\right) r_{1}
\end{aligned}
$$

where $\xi \in\left(r_{0}, r_{1}\right)$. Using Lemma 3.2 it is $t_{h}^{\prime}(\xi) \leq \varphi\left(\xi, t_{h}(\xi)\right)$, and hence

$$
0<t_{h}^{\prime}(\xi)-\varphi\left(\xi, t_{\rho}(\xi)\right) \leq \frac{\partial \varphi}{\partial t}(\xi, \theta)\left(t_{h}(\xi)-t_{\rho}(\xi)\right) .
$$

Combining (3.3) and 3.4) one gets a contradiction if $\frac{\partial \varphi}{\partial t}(\xi, \theta) \leq 0$. 


\section{DiSCUSSION AND CONCLUSIONS}

It is important to analyze the relationship of the condition (3.1) of the former lemma with the weak energy condition (wec). The latter requires $\epsilon>0, \epsilon+p_{r} \geq 0$ and $\epsilon+p_{t} \geq 0$. Here, there is no condition on the tangential pressure. If the radial pressure is positive, then (3.1) coincides with the remaining inequalities of wec, and is therefore weaker. In presence of tensions $\left(p_{r}<0\right)$, it places a lower bound on the radial stress which again coincides with that coming from wec unless $\epsilon>1 / 8 \pi R^{2}$, when the lower bound on $p_{r}$ must be explicitly required.

The condition stated is only sufficient. As a test-bed we can use the dust case, in which the exact solution is known in closed form and the nature of the singularities is known in full details [8] (a discussion of dust collapse in terms of existence/non existence of solutions for non-linear o.d.e. can be found in [9]).

The solution space for marginally bound collapse can be parameterized in terms of an integer ( $n$, say) giving the order of the first non-vanishing derivative of the initial density profile at the center ( since $p_{r}$ is zero, the center is the unique point that can be naked). One easily finds that, for $n=1$ and $n=2, \partial \varphi / \partial t$ is positive near the center, while for $n>2$ one has

$$
\frac{\partial \varphi}{\partial t}\left(r, t_{h}(r)\right)=-\frac{1}{r}\left(1-\beta_{n} r^{n-3}\right)+O\left(r^{n-2}\right)
$$

where $\beta_{n}$ is a positive quantity proportional to minus the first non vanishing derivative of the density at the center. Thus, the singularity is certainly covered for any $n>4$ and for $n=3$ if $\beta_{3}<1$. Actually, however, we know that the singularity is naked if $n$ equals one, if $n$ equals two, or if $n$ equals three but $\beta_{3}$ is greater than a positive value $\beta_{c}$ which in turn is greater than one [8] $\left(\beta_{c}=(26+15 \sqrt{3}) / 4\right)$. Therefore, there exists is a region of the solutions space in which blackholes still form, while our (thereby only sufficient) condition does not hold. Interestingly enough, this phenomenon occurs - at least in dust spacetimes - near to the transition of the critical parameter. Physically, it reflects the fact that the absence of apparent horizon formation prior to singularity does not necessarily implies nakedness [10].

The results of the present paper can be applied to all the matter models that admit regular initial data in spherical symmetry. For fluid sources, the space of all such solutions can be parameterized in terms of three functions, namely the initial density, the initial velocity, and the equation of state [11]. A physically viable formulation of a Cosmic Censorship theorem for fluid bodies would therefore rely in a classification of this solution space in terms of the final outcome of the collapse. In this context the theorem proved here can be used to characterize a "large" subset of this space, that contains only blackholes. Work in this direction is in progress.

Analyzing in full generality what happens at the "boundary" of this subset, i.e. when a transition from blackholes to naked singularities is expected to occur, looks, unfortunately, still a quite far objective.

\section{REFERENCES}

[1] R. Penrose, Nuovo Cimento 1252 (1969).

[2] P. S. Joshi, Global aspects in gravitation and cosmology, (Clarendon press, Oxford, 1993).

[3] G. Magli, Class. Quantum Grav. 153215 (1998).

[4] T. Harada, H. Iguchi and K. Nakao, Phys. Rev. D 58 R041502 (1998).

[5] Kudoh, H. Harada, T. Iguchi, H. Phys. Rev. D (3) (2000) 104016,

[6] S. Jhingan and G. Magli, Phys. Rev. D61 (2000) 124006

[7] F. I. Cooperstock, S. Jhingan, P. S. Joshi and T. P. Singh, Class. Quantum Grav. 142195 (1997).

[8] T. P. Singh and P. S. Joshi, Class. Quantum Grav. 13559 (1996).

[9] R. Giambo' and G. Magli, preprint.

[10] Jhingan, S. Joshi, P. S.; Singh, T. P. Class.. Quantum Grav. 13 (1996) 3057.

[11] Jhingan, S. and Magli, G., In Recent developments in General Relativity B. Casciaro, D. Fortunato, A. Masiello, M. Francaviglia ed., Springer Verlag (Berlin) (2000). 
SUFFICIENT CONDITION FOR BLACKHOLE FORMATION IN SPHERICAL GRAVITATIONAL COLLAPSE

Dipartimento di Matematica E Fisica

UNIVERSITÀ DI CAMERINO, ITALY

E-mail address: giambo@campus.unicam.it, giannoni@campus.unicam.it

DiPARTIMENTO Di MATEMATICA,

POLITECNICO Di MILANO, ITALY

E-mail address: magli@mate.polimi.it 